\title{
Chlorophyll $f$-driven photosynthesis in a cavernous cyanobacterium
}

\author{
Lars Behrendt ${ }^{1,2,3}$, Asker Brejnrod ${ }^{2}$, Martin Schliep ${ }^{4}$, Søren J Sørensen², \\ Anthony WD Larkum ${ }^{4}$ and Michael Kühl ${ }^{1,4}$ \\ ${ }^{1}$ Marine Biological Section, Department of Biology, University of Copenhagen, Helsingør, Denmark; ${ }^{2}$ Section \\ of Microbiology, Department of Biology, University of Copenhagen, Copenhagen, Denmark; ${ }^{3}$ Section for \\ Biomolecular Sciences, Department of Biology, University of Copenhagen, Copenhagen N, Denmark and \\ ${ }^{4}$ Plant Functional Biology and Climate Change Cluster, University of Technology Sydney, Sydney, New South \\ Wales, Australia
}

\begin{abstract}
Chlorophyll (Chl) $f$ is the most recently discovered chlorophyll and has only been found in cyanobacteria from wet environments. Although its structure and biophysical properties are resolved, the importance of $\mathrm{Chl} f$ as an accessory pigment in photosynthesis remains unresolved. We found $\mathrm{Chl} f$ in a cyanobacterium enriched from a cavernous environment and report the first example of $\mathrm{Chl} f$-supported oxygenic photosynthesis in cyanobacteria from such habitats. Pigment extraction, hyperspectral microscopy and transmission electron microscopy demonstrated the presence of $\mathrm{Chl} a$ and $f$ in unicellular cyanobacteria found in enrichment cultures. Amplicon sequencing indicated that all oxygenic phototrophs were related to $\mathrm{KC1}$, a $\mathrm{Chl}$-containing cyanobacterium previously isolated from an aquatic environment. Microsensor measurements on aggregates demonstrated oxygenic photosynthesis at $742 \mathrm{~nm}$ and less efficient photosynthesis under 768- and 777-nm light probably because of diminished overlap with the absorption spectrum of $\mathrm{Chl} f$ and other far-red absorbing pigments. Our findings suggest the importance of $\mathrm{Chl}$ f-containing cyanobacteria in terrestrial habitats.

The ISME Journal (2015) 9, 2108-2111; doi:10.1038/ismej.2015.14; published online 10 February 2015
\end{abstract}

The textbook concept that oxygenic phototrophs primarily use radiation in the visible range (400$700 \mathrm{~nm}$ ) has been challenged by several findings of unique cyanobacteria and chlorophylls (Chl) over the past two decades (Miyashita et al., 1996; Chen et al., 2010; Croce and van Amerongen, 2014) Unicellular cyanobacteria in the genus Acaryochloris primarily employ $\mathrm{Chl} d$ for oxygenic photosynthesis at $700-720 \mathrm{~nm}$ (Miyashita et al., 1996) and thrive in shaded habitats with low levels of visible light but replete of near-infrared radiation (NIR, > $700 \mathrm{~nm}$, Kühl et al., 2005; Behrendt et al., 2011, 2012). Furthermore, Chl $f$ was recently discovered in filamentous (Chen et al., 2010; Airs et al., 2014; Gan et al., 2014) and unicellular cyanobacteria (Miyashita et al., 2014), enabling light harvesting even further into the NIR region up to $\sim 740 \mathrm{~nm}$, often aided by employing additional far-red light-absorbing pigments such as Chl $d$ and phycobiliproteins (Gan et al., 2014). Whereas the biochemical structure (Willows et al., 2013) and

Correspondence: L Behrendt, Marine Biological Section, Department of Biology, University of Copenhagen, Strandpromenaden 5, Helsingør DK-3000, Denmark.

E-mail: Behrendt.1@gmail.com

Received 23 September 2014; revised 14 December 2014; accepted 22 December 2014; published online 10 February 2015 biophysical properties (Li et al., 2013; Tomo et al., 2014) of Chl $f$ have been studied in detail, the actual importance of this new chlorophyll for photosynthesis is hardly explored (Li et al., 2014).

Chlorophyll $f$ has been found in cyanobacteria originating from aquatic/wet environments: the filamentous Halomicronema hongdechloris from stromatolites in Australia (Chen et al., 2012), a unicellar morphotype (Strain KC1) from Lake Biwa in Japan (Akutsu et al., 2011; Miyashita et al., 2014) and a filamentous Leptolyngbya sp. strain (JSC-1, Gan et al., 2014) from a hot-spring and in a unicellular Chlorogloeopsis fritschii strain from rice paddies (Airs et al., 2014). In this study, we report on a unicellular Chl $f$-containing cyanobacterium originating from a wet cavernous habitat and demonstrate its capability of NIR-driven oxygenic photosynthesis. Enrichments of the new cyanobacterium were obtained from a dense dark green-blackish biofilm dominated by globular morphotypes of Nostocaceae growing on moist limestone outside Jenolan Caves, NSW, Australia. The sampling site was heavily shaded even during mid-day with low irradiance levels of 400- to 700 -nm light varying from 0.5 to $5 \mu \mathrm{mol}$ photons $\mathrm{m}^{-2} \mathrm{~s}^{-1}$. Biofilms were carefully scraped off the substratum and kept in shaded zip-lock bags in a moist atmosphere until further processing. 
Samples were then incubated at $28^{\circ} \mathrm{C}$ in a $\mathrm{f} / 2$ medium under NIR at $720 \mathrm{~nm}(\sim 10 \mu \mathrm{mol}$ photons $\mathrm{m}^{-2} \mathrm{~s}^{-1}$ ) yielding conspicuous green cell aggregates after several months of incubation. Repeated transfer of the aggregates into fresh medium resulted in a culture predominated by green cell clusters (Figure 1a), exhibiting orange-red fluorescence upon excitation with blue light (Figure 1b). Transmission electron microscopy revealed that the green clusters consisted of slightly elongated unicellular cyanobacteria $(\sim 1$ - to $2-\mu \mathrm{m}$ wide and $\sim 2$ - to $3-\mu \mathrm{m}$ long), with stacked thylakoids and embedded in a joint polymer matrix (Figure 1c). Hyperspectral microscopy (Kühl and Polerecky, 2008) of the clusters revealed distinct troughs in the transmission spectra at absorption maxima indicative of Chl $a(675-680 \mathrm{~nm})$ and Chl $f(\sim 720 \mathrm{~nm}$; Figure 1d, red line). In situ spectral irradiance measurements at the sampling site showed strong depletion of visible wavelengths in the 480- to 710-nm range (Figure 1d, gray line), whereas highest light levels were found in the near-infrared region of the solar spectrum at $710-900 \mathrm{~nm}$. The presence of Chl $a$ and $f$ was further confirmed in enrichment cultures using high-performance liquid chromatography-based pigment analysis (Figure 1e, Supplementary Figure S1), while no Chl $d$ was detected. In addition, weak spectral signatures of carotenoids and phycobilins, with absorption occurring at $\sim 495$ and $665 \mathrm{~nm}$, were evident in the hyperspectral data. Cyanobacteria, including those producing $\mathrm{Chl} d / f$, are known to actively remodel their pigment content in response to the available light spectrum (Stomp et al., 2007; Chen and Scheer, 2013; Gan et al., 2014) and Chl $d / f$ has almost exclusively been found in cyanobacteria grown under far-red light and not under visible light (Kühl et al., 2005; Chen et al., 2010; Airs et al., 2014; Gan et al., 2014; Li et al., 2014; Miyashita et al., 2014). Recent work describes this acclimation response as 'Far-Red Light photoacclimation' (FaRLiP), which, in strain JSC-1, comprises a global change in gene expression and structural remodeling of the PSII/PSI core proteins and phycobilisome constituents (Gan et al., 2014). The extent to which this arrangement results in optimized photosynthetic performance is only known for the NIR ( $=710 \mathrm{~nm}$ )-acclimated strain JSC-1, where exposure to wavelengths $>695 \mathrm{~nm}$ resulted in $40 \%$ higher $\mathrm{O}_{2}$ evolution rates as compared with cells that were previously adapted to red light $(645 \mathrm{~nm}$;
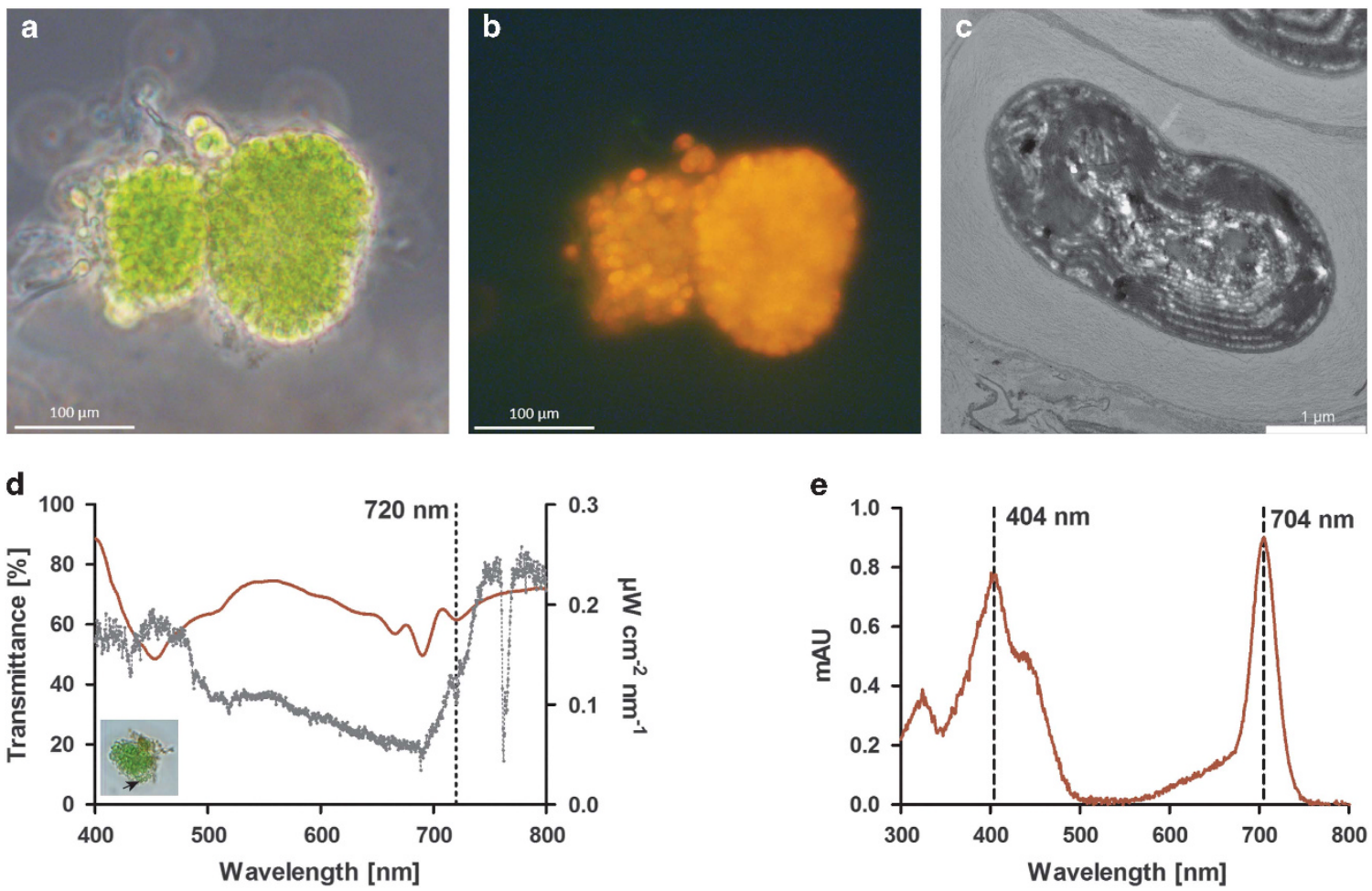

Figure 1 Imaging and pigment analysis of Chl $f$-containing cyanobacteria isolated from a cavernous low-light environment. (a) Representative bright field microscope image of cultured cells grown under $720 \mathrm{~nm}$ NIR. (b) Fluorescence image of the same cells as in a, excited at $450-490 \mathrm{~nm}$, with emission being detected at $>510 \mathrm{~nm}$. (c) Transmission electron microscopy of a Chl $f$-containing cyanobacterium with densely stacked thylakoid membranes. (d) Transmittance spectrum of cell aggregate determined by hyperspectral imaging (red line). Ambient light conditions at the site of isolation (gray line), as measured by a spectroradiometer. Note the Chl $f$-specific in vivo absorption at $\sim 720 \mathrm{~nm}$ in the transmittance spectrum (dotted line). Small insert picture denotes the cells and area of interest (black arrow) from which the spectrum was taken. (e) In vitro absorption spectrum of Chl $f$ extracted from enrichment cultures and analyzed via high-performance liquid chromatography. The two Chl f-specific absorption peaks (404 and $704 \mathrm{~nm}$ in acetone:MeOH solvent) are indicated. 

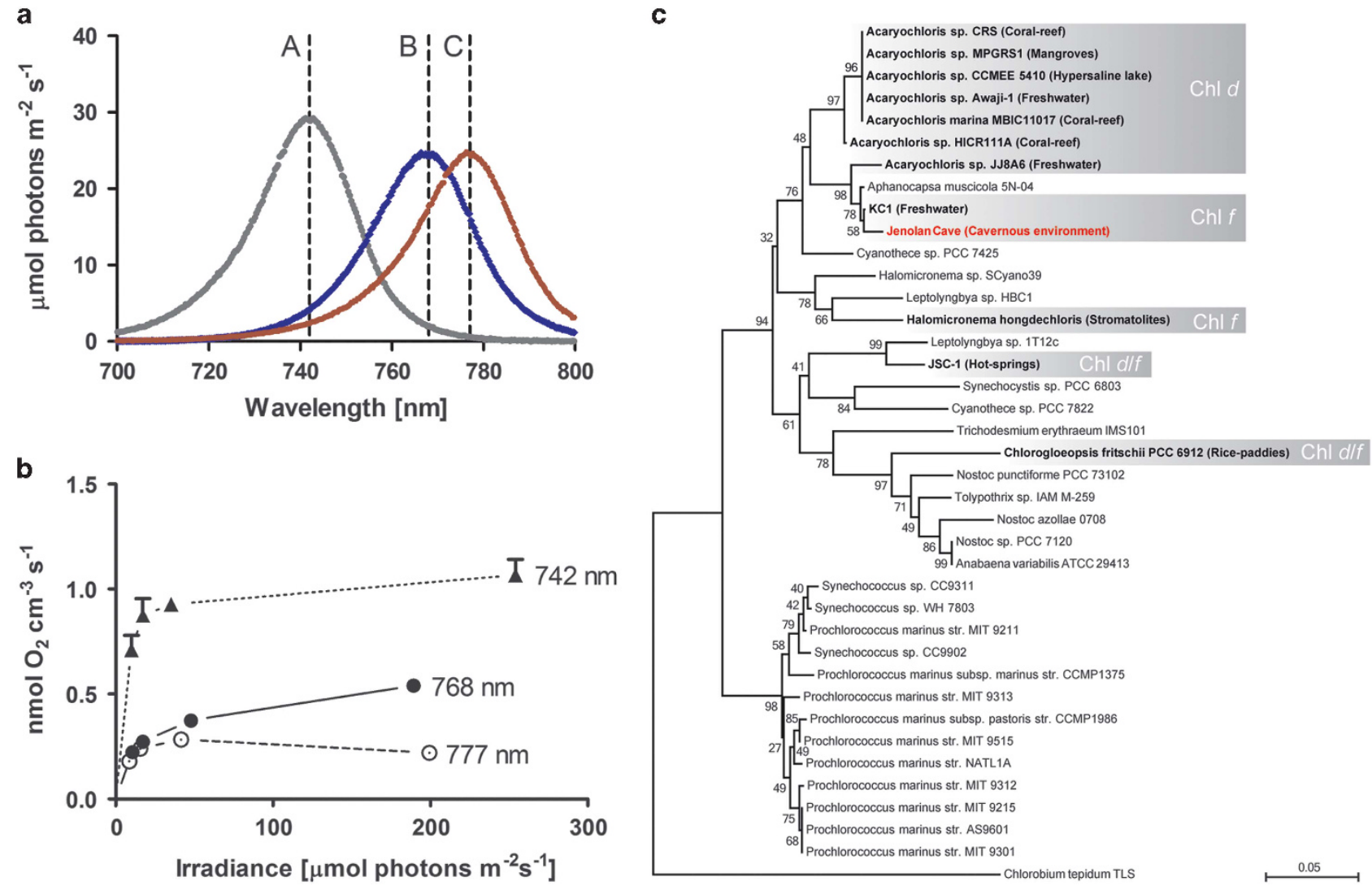

Figure 2 Taxonomic affiliation and $\mathrm{O}_{2}$ evolution of $\mathrm{Chl} f$-containing cells as determined by $\mathrm{O}_{2}$ microelectrode measurements and $16 \mathrm{~S}$ rDNA amplicon sequencing. (a) Emission spectra of narrow-band light-emitting diodes (LEDs) used in this study, with peak emissions at 742, 768 and $777 \mathrm{~nm}$ indicated by a-c, respectively. (b) Gross photosynthesis measured via an $\mathrm{O}_{2}$ microsensor placed in a clump of agarose-embedded Chl $f$-containing cells. Different NIR irradiance was administered by the LEDs in a and by altering the distance of the LEDs to the embedded cells. (c) Phylogenetic affiliation of known $\mathrm{Chl} f$ and/or Chl $d$-containing cyanobacteria (highlighted in gray) and their respective habitat/place of isolation. Taxonomy was determined by clustering all known oxygenic phototrophs found in enrichment cultures from this study (at order level) into a single OTU ( $=292$ bp length, see Supplementary Materials for details). Phylogeny was inferred using Maximum-likelihood in conjunction with the GTR + I + G nucleotide substitution model, tree stability was tested using bootstrapping with 100 replicates. The analysis involved 39 nucleotide sequences each truncated to a length of $292 \mathrm{bp}$. Here, the greensulphur bacterium Chlorobium tepidum TLS was chosen as the outgroup.

Gan et al., 2014). Yet the discrimination of actinic wavelengths and their relative effect on gross photosynthesis in Chl $f$-containing cells needs further investigation. Using an $\mathrm{O}_{2}$ microsensor and the light-dark shift method (Revsbech et al., 1983) on embedded Chl $f$-containing aggregates, we found maximal gross photosynthesis rates $\left(\sim 1.06 \mu \mathrm{mol} \mathrm{O}_{2}\right.$ $\mathrm{cm}^{-3} \mathrm{~s}^{-1}$ ) to occur at irradiances of $\sim 250 \mu \mathrm{mol}$ photons $\mathrm{m}^{-2} \mathrm{~s}^{-1}$ of $742 \mathrm{~nm}$ (half-bandwidth, HBW, $25 \mathrm{~nm}$, Figures $2 \mathrm{a}$ and b) with light saturation to occur very early at $\sim 35 \mu \mathrm{mol}$ photons $\mathrm{m}^{-2} \mathrm{~s}^{-1}$. Further redshifted actinic light, that is, $768 \mathrm{~nm}$ (HBW $28 \mathrm{~nm}$ ) and $777 \mathrm{~nm}$ (HBW $30 \mathrm{~nm}$ ), yielded lower $\mathrm{O}_{2}$ evolution rates, which, in all likelihood, are an effect of the diminished overlap with far-red light-absorbing pigments, including Chl $f$ (Figures 2a and b). As $\mathrm{O}_{2}$ evolution rates were measured on non-axenic cell aggregates, 16S rDNA amplicon sequencing was employed to determine the microbial diversity found within the enrichment culture. This revealed the presence of a variety of bacterial types, including anoxygenic phototrophs, yet all sequences for known oxygenic phototrophs in the data set $(\sim 9.3 \%$ of all reads on the order level, Supplementary Figure S2) formed a single operational taxonomic unit (OTU) closely affiliated with the Chl f-containing strain KC1 (Miyashita et al., 2014, Figure 2c).

This advocates that cells from our enrichment culture are related to KC1 cells and supports, in conjunction with further morphological-, physiological- and ultrastructural evidence, that Chl $f$ is extending the usable light spectrum for oxygenic photosynthesis in a cavernous low-light environment. Given the lifestyle and known habitats of recognized Chl $d / f$-producing cyanobacteria (Figure 2c), we propose that many, if not all, surface-associated cyanobacteria are intrinsically capable of producing far-red light-absorbing pigments and to actively employ them in oxygenic photosynthesis as a result of FaRLiP or similar, yet unknown, mechanisms.

\section{Conflict of Interest}

The authors declare no conflict of interest 


\section{Acknowledgements}

This study was supported by a grant from the Danish Research Council for Independent Research I Natural Sciences (MK). We thank Erik Trampe for assistance with high-performance liquid chromatography and hyperspectral analyses, Daniel Wangpraseurt and Milan Szabo for assistance with light measurements in the field, and Klaus Qvortrup for assistance with electron microscopy.

\section{Note added in proof}

Since the acceptance of this article, a survey of several cyanobacterial cultures from aquatic and terrestrial habitats has shown widespread occurrence of the ability to synthesize Chl $d$, Chl $f$, and modified phycobilins when incubated under far-red light, further corroborating our findings (Gan F, Shen G, Bryant DA. (2015). Occurrence of far-red light photoacclimation (FaRLiP) in diverse Cyanobacteria. Life 5: 4-24).

\section{References}

Airs RL, Temperton B, Sambles C, Farnham G, Skill SC, Llewellyn CA. (2014). Chlorophyll f and chlorophyll d are produced in the cyanobacterium Chlorogloeopsis fritschii when cultured under natural light and near-infrared radiation. FEBS Lett 588: 3770-3777.

Akutsu S, Fujinuma D, Furukawa H, Watanabe T. (2011). Pigment analysis of a chlorophyll $f$ - containing cyanobacterium strain KC1 isolated from Lake Biwa. Photomed Photobiol 33: 35-40.

Behrendt L, Larkum A, Norman A, Qvortrup K, Chen M, Ralph P et al. (2011). Endolithic chlorophyll $d$-containing phototrophs. ISME J 5: 1072-1076.

Behrendt L, Larkum A, Trampe E, Norman A, Sørensen S, Kühl M. (2012). Microbial diversity of biofilm communities in microniches associated with the didemnid ascidian Lissoclinum patella. ISME J 6: 1222-1237.

Chen M, Li Y, Birch D, Willows R. (2012). A cyanobacterium that contains chlorophyll $f$ - a red-absorbing photopigment. FEBS Lett 586: 3249-3254.
Chen M, Scheer H. (2013). Extending the limits of natural photosynthesis and implications for technical light harvesting. J Porphyrins Phthalocyanines 17: 1-15.

Chen M, Schliep M, Willows R, Cai Z-L, Neilan B, Scheer H. (2010). A red-shifted chlorophyll. Science 329: 1318-1319.

Croce R, van Amerongen H. (2014). Natural strategies for photosynthetic light harvesting. Nat Chem Biol 10: 492-501.

Gan F, Zhang S, Rockwell NC, Martin SS, Lagarias C, Bryant DA. (2014). Extensive remodeling of a cyanobacterial photosynthetic apparatus in far-red light. Science 345: 1312-1317.

Kühl M, Chen M, Ralph P, Schreiber U, Larkum AWD. (2005). A niche for cyanobacteria containing chlorophyll $d$. Nature 433: 820.

Kühl M, Polerecky L. (2008). Functional and structural imaging of phototrophic microbial communities and symbioses. Aquat Microb Ecol 53: 99-118.

Li Y, Cai Z, Chen M. (2013). Spectroscopic properties of chlorophyll $f$. J Phys Chem B 117: 11309-11317.

Li Y, Lin Y, Loughlin P, Chen M. (2014). Optimization and effects of different culture conditions on growth of Halomicronema hongdechloris-a filamentous cyanobacterium containing chlorophyll $f$. Front Plant Sci 25: 5-67.

Miyashita H, Ikemoto H, Kurano N, Adachi K, Chihara M, Miyachi S. (1996). Chlorophyll $d$ as a major pigment. Nature 383: 402.

Miyashita H, Ohkubo S, Hirohisa K, Yuhta S, Daisuke F, Daiki F et al. (2014). Discovery of Chlorophyll $d$ in Acaryochloris marina and Chlorophyll $f$ in a unicellular Cyanobacterium, Strain KC1, Isolated from Lake Biwa. J Phys Chem 4: 1-9.

Revsbech N, Jorgensen B, Blackburn T, Cohen Y. (1983). Microelectrode studies of the photosynthesis and $\mathrm{O}_{2}$ $\mathrm{H}_{2} \mathrm{~S}$, and $\mathrm{pH}$ profiles of a microbial mat. Limnol Oceanogr 28: 1062-1074.

Stomp M, Huisman J, Stal LJ, Matthijs HCP. (2007). Colorful niches of phototrophic microorganisms shaped by vibrations of the water molecule. ISME J 1: 271-282.

Tomo T, Shinoda T, Chen M, Allakhverdiev SI, Akimoto S. (2014). Energy transfer processes in chlorophyll $f$-containing cyanobacteria using time-resolved fluorescence spectroscopy on intact cells. Biochim Biophys Acta 1837: 1484-1489.

Willows R, Li Y, Scheer H, Chen M. (2013). Structure of chlorophyll f. Org Lett 15: 1588-1590.

Supplementary Information accompanies this paper on The ISME Journal website (http://www.nature.com/ismej) 